Outcomes of resected nonfunctional pancreatic neuroendocrine tumors : Do size and symptoms matter?

Sallinen, Ville

2015-12

Sallinen , V , Haglund , C \& Seppanen , H 2015 , ' Outcomes of resected nonfunctional pancreatic neuroendocrine tumors : Do size and symptoms matter? ' , Surgery , vol. 158 , no. 6 , pp. 1557-1564 . https://doi.org/10.1016/j.surg.2015.04.035

http://hdl.handle.net/10138/159093

https://doi.org/10.1016/j.surg.2015.04.035

publishedVersion

Downloaded from Helda, University of Helsinki institutional repository.

This is an electronic reprint of the original article.

This reprint may differ from the original in pagination and typographic detail.

Please cite the original version. 


\section{Outcomes of resected nonfunctional pancreatic neuroendocrine tumors: Do size and symptoms matter?}

Ville Sallinen, MD, PhD, Caj Haglund, MD, PhD, and Hanna Seppänen, MD, PhD, Helsinki, Finland

Background. Nonfunctional pancreatic neuroendocrine tumors (NF-PNETs) are rare tumors with highly variable outcome. Current guidelines recommend surveillance for small tumors $(\leq 2 \mathrm{~cm})$, but a scientific basis for such recommendation is scarce.

Methods. Patients who underwent surgery for NF-PNET during 2001-2013 were identified from a prospectively maintained database and reviewed retrospectively.

Results. Fifty-eight patients that had undergone an operative procedure for NF-PNET were identified. Forty-one patients ( $71 \%$ ) were symptomatic. Median size of the tumor was $2.5 \mathrm{~cm}$ (range $0.9-12.0 \mathrm{~cm}$ ). WHO 2010 grade was predictive of both overall-and disease-free survival $(\mathrm{P}<.001)$, whereas size alone was not. Twenty-four patients had a small NF-PNET $(\leq 2 \mathrm{~cm})$, of whom 16 were symptomatic and 8 asymptomatic. Seven patients with small symptomatic NF-PNETs showed signs of malignant behavior: 4 had lymph node metastases, 1 had liver metastases before surgery, 3 developed liver metastases, and 3 died of the disease. All 7 patients had either bile duct or pancreatic duct obstruction or both on preoperative imaging. On the contrary, patients with small asymptomatic NF-PNETs did not develop distant metastases nor died of disease.

Conclusion. The 2010 grading system from the World Health Organization can be used to predict survival. Symptomatic small NF-PNETs that caused bile and/or pancreatic duct obstruction had poor outcome. In contrast, asymptomatic small NF-PNETs seem to have benign course, and are candidates for surveillance. (Surgery 2015;158:1556-63.)

From the Department of Abdominal Surgery, University of Helsinki, Helsinki University Hospital, Helsinki, Finland

Pancreatic neuroendocrine tumors (PNETs) are rare neoplasms with an increasing incidence of 0.43 per 100,000 people, which is more than double the incidence a few decades ago. ${ }^{1}$ Functional PNETs excrete active hormones, which cause specific endocrine symptoms, whereas most PNETs are nonfunctional pancreatic neuroendocrine tumors (NF-PNET). ${ }^{1}$ Functional PNETs usually require surgery to control the symptoms from the excreted hormone. The decision to operate on NF-PNETs is more difficult, especially as many

C. Haglund and H. Seppänen contributed equally to this work. This study was funded by Vatsatautien tutkimussäätiö Foundation, Mary and Georg Ehrnrooth's Foundation, and governmental competitive funds (EVO).

Accepted for publication April 29, 2015.

Reprint requests: Ville Sallinen, MD, PhD, Department of Abdominal Surgery, Helsinki University Central Hospital, Haartmaninkatu 4, 00029 HUS, Finland. E-mail: ville.sallinen@ helsinki.fi.

0039-6060/\$ - see front matter

(c) 2015 Elsevier Inc. All rights reserved.

http://dx.doi.org/10.1016/j.surg.2015.04.035
NF-PNETs are found incidentally. ${ }^{2}$ Multiple neuroendocrine neoplasia type 1 (MEN1) and Von Hippel-Lindau (VHL) are syndromes that predispose patients for PNETs, and these patients may harbor multiple PNETs.

For help in decision making, World Health Organization (WHO) has developed a 3-stage classification for PNETs based on their mitotic activity to predict the aggressiveness of the tumors. ${ }^{3,4}$ The dilemma in treating patients with NF-PNET arises from the high morbidity caused by pancreatic surgery, which needs to be balanced against the aggressiveness of the tumor and patient's comorbidities. Furthermore, the WHO classification requires histopathologic analysis of the tumor, which might not be possible preoperatively, especially in small tumors. Consequently, many guidelines have used size to determine appropriate treatment. Size larger than $2 \mathrm{~cm}$ is used as a cut-off between surveillance and operative treatment of NF-PNETs. ${ }^{1,5,6}$ The aim of this study was to evaluate the significance of size and symptoms of NF-PNETs on metastases, progression, and survival. 


\section{PATIENTS AND METHODS}

Patients were identified from a prospectively maintained database, which comprises patients who underwent pancreatic surgery during 20002013. Medical records, radiologic reports, and histopathologic findings were reviewed retrospectively. Patients whose postoperative histopathologic diagnosis was PNET were included, and their charts were retrieved. Patients who had symptoms related to excreted hormone (functional PNETs) were excluded. Follow-up data were obtained from the community hospitals, which carried out the follow-up visits. End of follow-up was defined as a last contact marked in the patient records, or date of death obtained from Population Register Center. Curative intent surgery was defined as surgery that aimed to remove all tumor tissue and included both enucleation and formal resections but no R2resections. Resection of the primary tumor in the presence of metastases or debulking surgery was not considered as curative intent surgery.

The Charlson comorbidity index was used to classify patient's comorbidities. Pancreatic fistula and delayed gastric emptying were graded by use of the definitions by the International Study Group of Pancreatic Surgery. ${ }^{7,8}$ Clavien-Dindo classification was used to grade all complications. WHO 2010 classification was used to grade the tumors. ${ }^{3}$ Survival analyses using Kaplan-Meier and log-rank test were done using SPSS 20.0 (IBM, Armonk, $\mathrm{NY}$ ). The study was approved by the institutional review board and the local ethics committee.

\section{RESULTS}

Patients. Fifty-eight consecutive patients who underwent resection for NF-PNET during 20012013 were identified from the database. Baseline characteristics of the patients are shown in Table I. Median age was 61 years (range 33-79) and 27 $(47 \%)$ were men. Ten patients $(17 \%)$ had an underlying syndrome making them susceptible to PNETs (9 MEN1 and 1 VHL). Forty-one patients (71\%) had symptoms that lead to the investigations discovering NF-PNET, whereas in 17 patients (29\%) the tumor was found incidentally at imaging studies made for other reasons. The most common symptom was abdominal pain $(n=25,43 \%)$, followed by jaundice $(n=10,17 \%)$. Of the small tumors (2 cm or less), 8 (33\%) were asymptomatic, $3(13 \%)$ had weight loss, $5(21 \%)$ had jaundice, and $11(46 \%)$ had abdominal pain. Tumors were located in the head in 21 patients $(36 \%)$, the body in 11 patients (19\%), and the tail in 19 (33\%) patients without any clear dominance of
Table I. Baseline characteristic of the patients and tumors

\begin{tabular}{lr}
\hline \multicolumn{1}{c}{ Total, $\mathrm{n}=58$} & $\mathrm{n}(\%)$ \\
\hline Age, y, median (range) & $60.5(33-79)$ \\
Sex, M/F, $n(\%)$ & $27(47) / 31(53)$ \\
Charlson comorbidity index, & $3(2-8)$ \\
$\quad$ median (range) & \\
Syndrome, $n(\%)$ & $9(16)$ \\
MEN1 & $1(2)$ \\
VHL & $2(3)$ \\
HNPCC & \\
Symptom, $n(\%) *$ & $25(43)$ \\
Pain & $10(17)$ \\
Jaundice & $11(19)$ \\
Weight loss & $2(3)$ \\
Palpable mass & $17(29)$ \\
Asymptomatic & \\
Tumor localization, $n(\%)$ & $21(36)$ \\
Head & $11(19)$ \\
Body & $19(33)$ \\
Tail & $7(12)$ \\
Multiple & $2.5(0.9-12.0)$ \\
Tumor size, cm, median (range) & \\
*Total percentage greater than 100\% as some patients presented with \\
several symptoms. \\
HNPCC, Hereditary nonpolyposis colorectal \\
Lynch syndrome; MEN1, multiple endocrine \\
von Hippel-Lindau syndrome. & \\
&
\end{tabular}

any of the locations. Multiple NF-PNETs were found in 7 patients (12\%), of whom 5 had MEN1 syndrome, 1 VHL syndrome, and 1 hereditary nonpolyposis colorectal cancer syndrome. Among these patients, only the patient with VHLsyndrome was symptomatic (pain).

Surgery. Forty-six patients $(79 \%)$ underwent formal pancreatic resection, whereas 9 patients $(16 \%)$ had their tumor enucleated (Table II). Median size of enucleated tumors was $17 \mathrm{~mm}$ (range $10-23 \mathrm{~mm}$ ). Two patients (3\%) underwent simultaneous distal pancreatectomy and enucleation of a tumor located in the head. Total pancreatectomy was performed on one patient $(2 \%)$. Majority of the cases were operated by open approach (45 patients, $78 \%$ ), whereas 15 patients (26\%) were approached laparoscopically. Four patients undergoing a laparoscopic approach initially were converted to open surgery (27\%). Lymphadenectomy was performed on 35 patients $(60 \%)$. Overall, 44 patients $(76 \%)$ underwent curative intent surgery, whereas $14(24 \%)$ underwent a debulking procedure or resection of the primary tumor in spite of metastases. Most of the patients (10 of 14) who underwent debulking operation or primary tumor resection had a large tumor $(\geq 4 \mathrm{~cm})$. 
Table II. Operative strategies and short-term morbidity

\begin{tabular}{|c|c|c|c|c|}
\hline & & & & $\mathrm{n}(\%)$ \\
\hline \multicolumn{5}{|l|}{ Operative procedure } \\
\hline Pancreaticoduodenectomy & & & & $20(35)$ \\
\hline Median pancreatectomy & & & & $1(2)$ \\
\hline Distal pancreatectomy* & & & & $26(45)$ \\
\hline Uncinate process resection & & & & $1(2)$ \\
\hline Enucleation* & & & & $11(19)$ \\
\hline Total pancreatectomy & & & & $1(2)$ \\
\hline \multicolumn{5}{|l|}{ Approach } \\
\hline Open surgery & & & & $45(78)$ \\
\hline Laparoscopic converted to open s & irgery & & & $4(7)$ \\
\hline \multirow[t]{2}{*}{ Laparoscopic } & & & & $9(16)$ \\
\hline & Head resection, $\mathrm{n}=21$ & Distal resection, ${ }^{*} \mathrm{n}=26$ & Enucleation, ${ }^{*} \mathrm{n}=11$ & $A l l, \dagger \mathrm{n}=58$ \\
\hline Complications, all & $13(62)$ & $16(62)$ & $8(73)$ & $37(64)$ \\
\hline \multicolumn{5}{|l|}{ Type } \\
\hline \multicolumn{5}{|l|}{ Pancreatic fistula } \\
\hline Grade A & $3(14)$ & $9(35)$ & $3(27)$ & $14(24)$ \\
\hline Grade B & $1(5)$ & $1(4)$ & $1(9)$ & $4(7)$ \\
\hline Grade $\mathrm{C}$ & $1(5)$ & $5(19)$ & $3(27)$ & $8(14)$ \\
\hline Biliary leakage & $3(14)$ & - & - & $3(5)$ \\
\hline Pulmonary embolism & $1(5)$ & 0 & 0 & $1(2)$ \\
\hline \multicolumn{5}{|l|}{ Delayed gastric emptying } \\
\hline Grade A & 0 & $1(4)$ & 0 & $1(2)$ \\
\hline Grade B & $3(14)$ & 0 & 0 & $3(5)$ \\
\hline Grade $\mathrm{C}$ & $3(14)$ & 0 & 0 & $3(5)$ \\
\hline Wound infection & $1(5)$ & 0 & 0 & $1(2)$ \\
\hline Intra-abdominal abscess & $4(19)$ & $2(8)$ & 0 & $7(12)$ \\
\hline Pneumonia & $2(10)$ & $1(4)$ & 0 & $3(5)$ \\
\hline \multicolumn{5}{|l|}{ Severity } \\
\hline None & $8(38)$ & $10(39)$ & $3(27)$ & $21(36)$ \\
\hline Clavien-Dindo 1-2 & $5(29)$ & $11(42)$ & $4(32)$ & $20(34)$ \\
\hline Clavien-Dindo 3 & $4(19)$ & $4(15)$ & $4(36)$ & $12(21)$ \\
\hline Clavien-Dindo 4 & $2(20)$ & $1(4)$ & 0 & $4(7)$ \\
\hline Clavien-Dindo 5 (death) & $1(5)$ & 0 & 0 & $1(2)$ \\
\hline Hospital stay, days, median (range) & $15(7-36)$ & $8(5-64)$ & $5(3-11)$ & $8(3-64)$ \\
\hline
\end{tabular}

*Two patients underwent simultaneous distal pancreatectomy and enucleation.

$\dagger$ Includes also complications for median $(n=1)$ and total $(n=1)$ pancreatectomy.

Thirty-seven patients $(64 \%)$ had complications after surgery (Table II). Clinically relevant pancreatic fistula (grade $\mathrm{B} / \mathrm{C}$ ) was the most common complication (12 patients; 21\%), followed by delayed gastric emptying $(n=7 ; 12 \%)$. One patient $(2 \%)$ died after surgery. Eight of nine enucleated tumors were WHO 2010 grade 1, and one was grade 2. None of these patients had recurrence or died of NF-PNET during follow-up.

Tumor characteristics. Fifty patients $(86 \%)$ had a single NF-PNET, 3 patients $(5 \%)$ had 2 NFPNETs, and 5 patients $(9 \%)$ had 3 synchronous NF-PNETs. Median size of the tumor was $2.5 \mathrm{~cm}$ (range 0.9-12.0). Twenty-four patients (41\%) had NF-PNET of $2 \mathrm{~cm}$ or less. For analyses, tumors were divided into 3 groups (Table III): small (diameter $2 \mathrm{~cm}$ or less), medium $(2-4 \mathrm{~cm})$, and large $(4 \mathrm{~cm}$ or larger). Metastatic lymph nodes were found in 4 patients $(17 \%)$ with tumor size $2 \mathrm{~cm}$ or less. The smallest tumor to have lymph node metastases was $15 \mathrm{~mm}$. Medium and large tumors had metastatic lymph nodes in $10(59 \%)$ and 9 patients $(53 \%)$, respectively.

Three of 22 patients $(14 \%)$ with a small tumor who had underwent a curative intent resection for local disease had recurrence during follow-up. Median time to recurrence was 12 months. Primarily, 1 had WHO 2010 grade 2 and 2 grade 3 tumors. The smallest tumor to recur after curative intent resection was $14 \mathrm{~mm}$. Medium and large tumors recurred in 5 of 13 (38\%) and 3 of 7 (43\%) 
Table III. Tumor and prognosis characteristics in relation to size of the tumor

\begin{tabular}{|c|c|c|c|c|}
\hline & $\begin{array}{c}\text { Small } \leq 2 \mathrm{~cm} \\
\mathrm{n}=24\end{array}$ & $\begin{array}{c}\text { Medium } \\
2>4 \mathrm{~cm} \\
\mathrm{n}=17\end{array}$ & $\begin{array}{c}\text { Large } \geq 4 \mathrm{~cm}, \\
\mathrm{n}=17\end{array}$ & $A l l, \mathrm{n}=58$ \\
\hline Patients with lymph nodes examined & $9(38)$ & $14(82)$ & $12(71)$ & $35(60)$ \\
\hline \multicolumn{5}{|l|}{ WHO 2010 grade } \\
\hline G1 & $14(58)$ & $3(18)$ & $1(6)$ & $18(31)$ \\
\hline G2 & $6(25)$ & $12(71)$ & $12(71)$ & $30(52)$ \\
\hline G3 & $4(17)$ & $2(12)$ & $4(24)$ & $10(17)$ \\
\hline Neural invasion & $3(13)$ & $8(47)$ & $5(29)$ & $16(28)$ \\
\hline Vascular invasion & $5(21)$ & $9(53)$ & $9(53)$ & $23(40)$ \\
\hline Necrosis & $1(4)$ & $4(24)$ & $3(18)$ & $8(14)$ \\
\hline $\begin{array}{l}\text { Time from diagnosis to surgery, months, } \\
\text { median (IQR) }\end{array}$ & $3(2-6)$ & $3(2-8)$ & $3(2-7)$ & $3(2-6)$ \\
\hline \multicolumn{5}{|l|}{ Distant metastases before surgery } \\
\hline Lymph node & 0 & $1(6)$ & 0 & $1(2)$ \\
\hline Liver & $1(4)$ & $3(18)$ & $7(41)$ & $11(19)$ \\
\hline Lung & 0 & 0 & $2(12)$ & $2(3)$ \\
\hline Multiple distant location & 0 & 0 & $1(6)$ & $1(2)$ \\
\hline Neoadjuvant therapy & $1(4)$ & $3(18)$ & $4(24)$ & $8(14)$ \\
\hline Adjuvant therapy & $6(25)$ & $9(53)$ & $9(53)$ & $24(41)$ \\
\hline Curative intent & $23(96)$ & $14(82)$ & $7(41)$ & $44(76)$ \\
\hline Debulking/primary tumor resection & $1(4)$ & $3(18)$ & $10(59)$ & $14(24)$ \\
\hline Recurrence after curative intent resection & $3 / 22(14)$ & $5 / 13(38)$ & $3 / 7(43)$ & $12 / 42(29)$ \\
\hline \multicolumn{5}{|l|}{ Location of recurrence* } \\
\hline Local & 0 & $1(8)$ & $1(14)$ & $3(5)$ \\
\hline Liver & $3(14)$ & $5(38)$ & $2(29)$ & $10(24)$ \\
\hline Lymph node & 0 & $1(8)$ & 0 & $1(2)$ \\
\hline Time to recurrence, mo, median (range) & 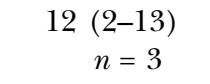 & $\begin{array}{c}24(3-45) \\
n=5\end{array}$ & $\begin{array}{c}27(20-49) \\
n=3\end{array}$ & $\begin{array}{c}17(2-49) \\
n=12\end{array}$ \\
\hline \multicolumn{5}{|l|}{ Metastasectomy } \\
\hline Liver resection & $1(4)$ & $3(18)$ & $6(35)$ & $10(17)$ \\
\hline Pancreatic resection & 0 & $1(6)$ & 0 & $1(2)$ \\
\hline Disease-specific death & $3(13)$ & 0 & $6(35)$ & $9(16)$ \\
\hline Follow-up, mo, median (range) & $27(1-131)$ & $20(0-109)$ & $22(0-91)$ & $24(0-131)$ \\
\hline
\end{tabular}

*Same patient may have recurrence in several locations.

$I Q R$, Interquartile range; $W H O$, World Health Organization.

patients, respectively, after curative intent resection for local disease.

One patient with a small primary tumor had liver metastases at the time of the diagnosis, and another patient with a small primary tumor underwent only debulking due to local infiltration. Three patients $(13 \%)$ died because of progression of a small NF-PNET. None of these patients had MEN1 or VHL syndrome and all had underwent pancreaticoduodenectomy. The smallest tumor to cause NF-PNET-specific death was $15 \mathrm{~mm}$. None of the medium size NF-PNETs caused death during follow-up, whereas $6(35 \%)$ patients with a large NF-PNET died of disease.

Effect of WHO 2010 grade and tumor size on survival and recurrence. WHO 2010 grade was highly correlated with both overall and disease-free survival (Table IV, Fig). There was no diseasespecific mortality among patients with grade 1 tumors, whereas grade 2 tumors were associated with a $7 \%$ mortality (2 patients) and grade 3 tumors with a $70 \%$ mortality (7 patients). Because WHO 2010 grade is seldom known preoperatively, tumor size is used in decision making. Surprisingly, size did not correlate with overall or disease-free survival (Table IV, Fig). In overall survival analysis, patients with a 2- to 4-cm tumor had a better prognosis compared with those with small $(2 \mathrm{~cm}$ or less) or large (over $4 \mathrm{~cm}$ ) tumor (Fig). There was no statistical difference in the disease-free survival in patients with tumors of different sizes (Fig).

Small $(\leq 2 \mathrm{~cm})$ WHO 2010 grade 1 tumors. Patients with small WHO 2010 grade 1 tumors are of special interest, because these tumors are 
Table IV. Tumor and prognosis characteristics based on WHO 2010 grade

\begin{tabular}{|c|c|c|c|}
\hline & $G 1, \mathrm{n}=18$ & $G 2, \mathrm{n}=30$ & $G 3, \mathrm{n}=10$ \\
\hline Lymphadenectomy performed & $3(17)$ & $23(77)$ & $9(90)$ \\
\hline Metastatic lymph nodes found & $3(17)$ & $14(47)$ & $6(60)$ \\
\hline Neural invasion & $2(11)$ & $8(27)$ & $7(70)$ \\
\hline Vascular invasion & $4(22)$ & $13(43)$ & $7(70)$ \\
\hline Necrosis & 0 & $3(10)$ & $5(50)$ \\
\hline \multicolumn{4}{|l|}{ Distant metastases before surgery } \\
\hline Lymph node & $1(6)$ & 0 & 0 \\
\hline Liver & 0 & $8(27)$ & $3(30)$ \\
\hline Lung & 0 & 0 & $2(20)$ \\
\hline Multiple location & 0 & $1(3)$ & 0 \\
\hline \multicolumn{4}{|l|}{ Resection margins } \\
\hline Curative intent resection & $17(94)$ & $22(73)$ & $5(50)$ \\
\hline Debulking/primary tumor resection & $1(6)$ & $8(27)$ & $5(50)$ \\
\hline Recurrence after curative intent resection for local disease & 0 & $8(38)$ & $3(75)$ \\
\hline \multicolumn{4}{|l|}{ Type } \\
\hline Local & 0 & $1(13)$ & 0 \\
\hline Liver & 0 & $5(63)$ & $3(100)$ \\
\hline Liver + lymph node & 0 & $1(13)$ & 0 \\
\hline Liver + local & 0 & $1(13)$ & 0 \\
\hline \multirow[t]{2}{*}{ Lung } & 0 & 0 & 0 \\
\hline & $n=17$ & $n=21$ & $n=4$ \\
\hline Time to recurrence, mo, median (range) & - & $25(2-49)$ & $15(1-13)$ \\
\hline Disease-specific death & 0 & $2(7)$ & $7(70)$ \\
\hline
\end{tabular}

WHO, World Health Organization.

considered to be benign. There were 14 patients with grade 1 NF-PNET of size $2 \mathrm{~cm}$ or less. None of the patients had metastases before surgery. Seven patients $(50 \%)$ underwent an enucleation procedure, whereas 6 patients $(43 \%)$ underwent a formal resection (1 median pancreatectomy, 5 distal pancreatectomies). Furthermore, 1 patient (7\%) underwent a simultaneous distal pancreatectomy and enucleation procedure. Lymph nodes were examined in 1 patient (7\%), and 3 metastatic lymph nodes were found. One patient developed a ductal adenocarcinoma in the remnant pancreas 10 months after distal pancreatectomy. None of the patients had recurrence or died of NF-PNET.

Symptomatic versus asymptomatic small $(2 \mathrm{~cm}$ or less) tumors. There were 17 patients without any symptoms of their NF-PNETs. Eight of these patients had small $(2 \mathrm{~cm}$ or less) local asymptomatic tumor (Table V). None of the small asymptomatic tumors developed distant recurrence in the follow-up or caused mortality (Table V). In contrast, 7 patients of 16 with symptomatic small NF-PNETs had signs of malignant behaviour: NFPNET recurred in liver in 3 of these patients, 4 patients had metastatic lymph nodes, 1 patient had liver metastases before surgery, and 3 patients died of the disease. Three of these 7 patients had bile duct obstruction, 2 had bile and pancreatic duct obstruction, and 2 had only pancreatic duct obstruction. One patient with bile and pancreatic duct obstruction had also suspicion of metastatic lymph nodes in the preoperative computed tomography scan.

\section{DISCUSSION}

We report here 58 consecutive patients operated for NF-PNETs at our institution during 2001-2013. The WHO 2010 classification was found to predict both overall and disease-free survival. Tumor size alone did not predict survival, but a small portion of small $(2 \mathrm{~cm}$ or less) tumors showed malignant behavior. Subgroup analyses on these patients revealed that the worse outcomes were related exclusively to symptomatic patients, of whom $25 \%$ had metastatic lymph nodes, $19 \%$ recurred, and $19 \%$ died of disease. Furthermore, all of these patients who had poor outcome had either bile or pancreatic duct obstruction or both in preoperative imaging. Asymptomatic patients with small NFPNETs had good prognosis. None of the patients with small asymptomatic NF-PNET developed distant metastases nor died of the disease.

It is evident that NF-PNET is a disease entity with a broad spectrum of clinical behavior without clear cut-off for a benign disease. Even though the WHO 2010 classification shows good prognostic 
A

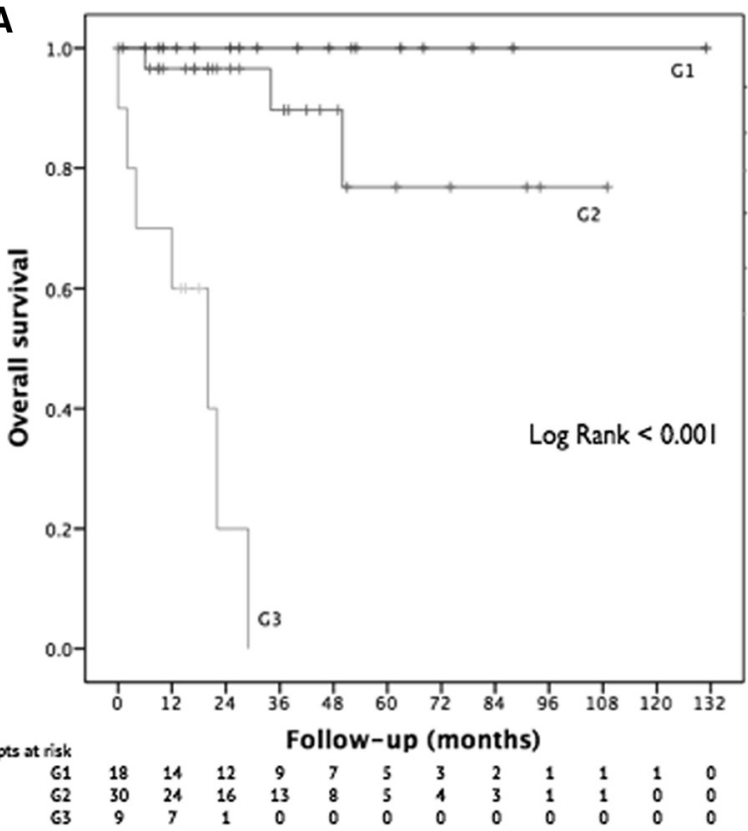

C

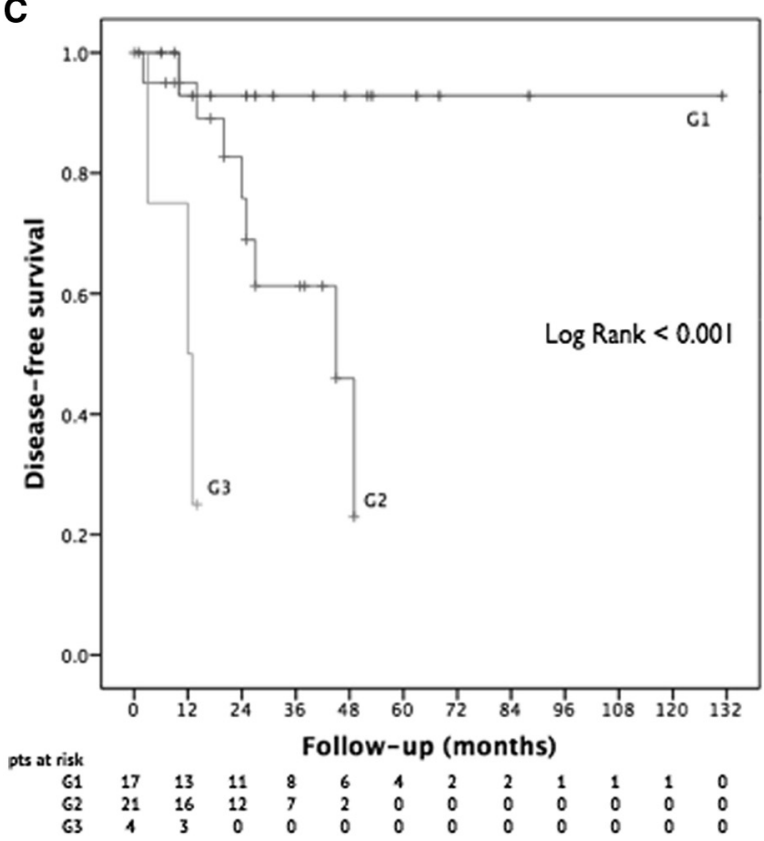

B

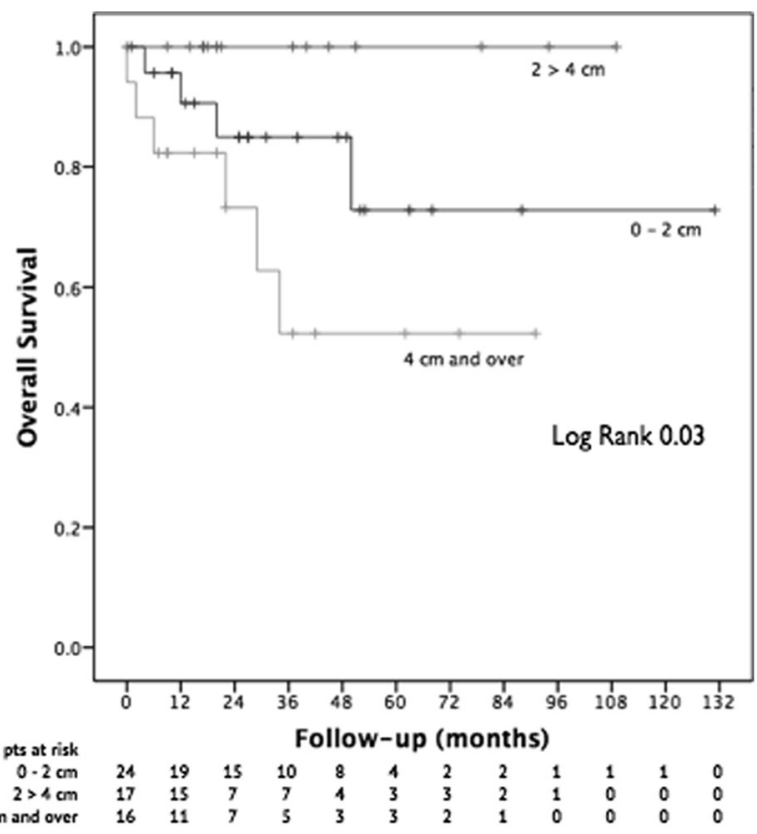

D

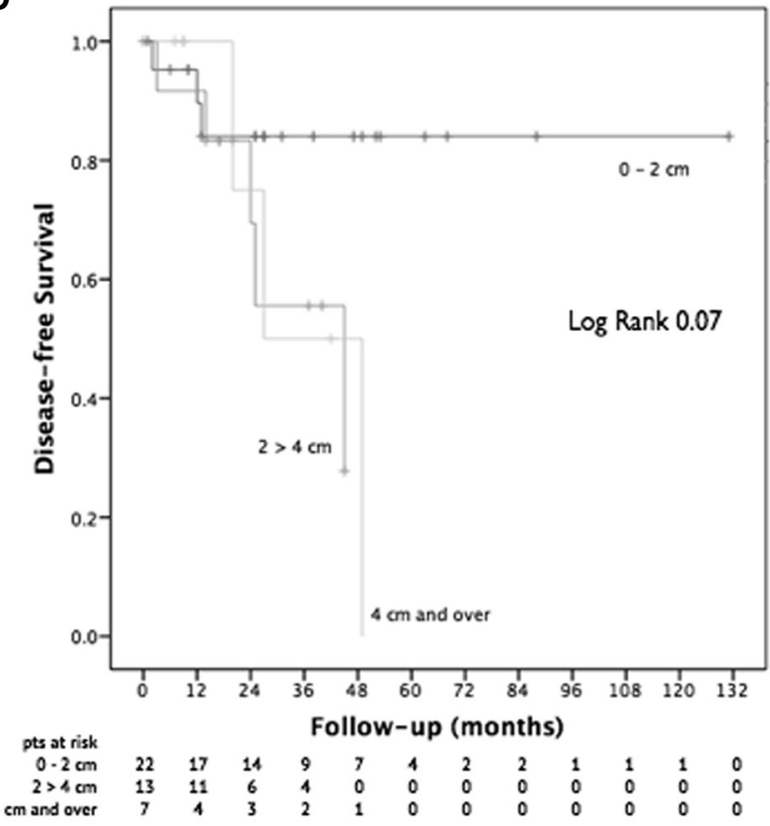

Fig. (A) Overall survival after NF-PNET resection stratified by WHO 2010 grade. (B) Overall survival after NF-PNET resection stratified by tumor size. (C) Disease-free survival after curative intent surgery for local disease stratified by WHO 2010 grade. (D) Disease-free survival after curative intent surgery for local disease stratified by tumor size.

value, the grade may not be available preoperatively. These findings demonstrate that size alone cannot differentiate whether a small $(2 \mathrm{~cm}$ or less) NF-PNETs is benign or malignant, and other variables needs to be taken account as well (symptoms, bile and/or pancreatic duct obstruction, and grade if available).

Similarly, Haynes et $\mathrm{al}^{9}$ reported 39 patients with small $(2 \mathrm{~cm}$ or less) NF-PNETs, of which 3 patients $(8 \%)$ had late metastases or recurrence and died of disease. Birnbaum et $\mathrm{al}^{2}$ showed that 7 out of 50 small NF-PNETs (14\%) had metastatic lymph nodes, which is similar to our finding $(13 \%)$.

Only $30 \%$ of our patients were asymptomatic, and the majority had symptoms possibly associated with the tumor. In fact, this is in contrast to many earlier publications, were the majority of patients 
Table V. Tumor and prognosis characteristics in relation to symptoms

\begin{tabular}{|c|c|c|c|}
\hline & $\begin{array}{l}\text { Symptomatic, } \\
\mathrm{n}=41\end{array}$ & $\begin{array}{c}\text { Asymptomatic, } \\
\mathrm{n}=17\end{array}$ & $\begin{array}{c}\text { Small }(\leq 2 \mathrm{~cm}) \text {, asymptomatic } \\
\text { and without metastases } \\
\text { before surgery, } \mathrm{n}=8\end{array}$ \\
\hline Patients with lymph nodes examined & $29(71)$ & $6(35)$ & 0 \\
\hline Patients with metastatic lymph nodes found & $18(44)$ & $5(29)$ & 0 \\
\hline \multicolumn{4}{|l|}{ WHO 2010 grade } \\
\hline G1 & $10(24)$ & $8(47)$ & $6(75)$ \\
\hline G2 & $22(54)$ & $8(47)$ & $2(25)$ \\
\hline G3 & $9(22)$ & $1(6)$ & 0 \\
\hline Neural invasion & $16(39)$ & $1(6)$ & 0 \\
\hline Vascular invasion & $21(51)$ & $3(18)$ & 0 \\
\hline Necrosis & $6(15)$ & $2(12)$ & 0 \\
\hline Time from diagnosis to surgery, months, median (IQR) & $2(1-4)$ & $5(2-16)$ & $11(2-18)$ \\
\hline \multicolumn{4}{|l|}{ Distant metastases before surgery } \\
\hline Lymph node & $2(5)$ & 0 & 0 \\
\hline Liver & $10(24)$ & $1(6)$ & 0 \\
\hline Lung & $1(2)$ & $1(6)$ & 0 \\
\hline Multiple distant location & $1(2)$ & 0 & 0 \\
\hline Neoadjuvant therapy & $7(17)$ & $1(6)$ & $1(13)$ \\
\hline Adjuvant therapy & $20(49)$ & $4(24)$ & $2(25)$ \\
\hline Curative intent & $29(71)$ & $15(88)$ & $8(100)$ \\
\hline Debulking/primary tumor resection & $12(29)$ & $2(12)$ & 0 \\
\hline Recurrence after curative intent resection & $8 / 29(28)$ & $2 / 15(13)$ & 0 \\
\hline \multicolumn{4}{|l|}{ Location of recurrence* } \\
\hline Local & $1(3)$ & $1(7)$ & 0 \\
\hline Liver & $9(31)$ & $2(13)$ & 0 \\
\hline Lymph node & $1(3)$ & 0 & 0 \\
\hline Time to recurrence, mo, median (range) & $14(6-24)$ & $36(27-45)$ & - \\
\hline \multicolumn{4}{|l|}{ Metastasectomy } \\
\hline Liver resection & $5(12)$ & $1(6)$ & 0 \\
\hline Pancreatic resection & 0 & $1(6)$ & 0 \\
\hline Disease-specific death & $8(20)$ & $1(6)$ & 0 \\
\hline
\end{tabular}

*Same patient may have recurrence in several locations.

$I Q R$, Interquartile range; $W H O$, World Health Organization.

undergoing surgery are asymptomatic. ${ }^{2,9-11}$ In our material, only patients whose tumor was revealed in a computed tomography scan performed for completely unrelated indications were classified as asymptomatic. In any case, the decision to operate on symptomatic patients is relatively straightforward compared with the ones that are asymptomatic. Incidental NF-PNETs have been associated with less aggressive features compared with symptomatic tumors. ${ }^{2}$ Yet, the reported 5and 10-year survival rates for symptomatic and asymptomatic NF-PNETs have been similar. $^{9}$ Although symptomatic NF-PNETs do not possess problems whether to resect them, we included them in the analyses to show that even small NFPNETs may behave aggressively. Small asymptomatic NF-PNETs showed relatively benign course, and none of the patients had distant recurrence or died of the disease.
The optimal operative strategy for NF-PNETs is debatable, especially concerning small NF-PNETs. Formal pancreatic resections (pancreaticoduodenectomy and distal resection) with lymphadenectomy are associated with significant morbidity and mortality as well as poor functional long-term outcome. This needs to be balanced against the relatively benign course of the majority of small NF-PNETs. In our material, 11 patients (19\%) underwent enucleation, whereas 49 patients (84\%) underwent a formal pancreatic resection (two patients underwent simultaneous distal pancreatectomy and enucleation). Rate of complications was greater in the enucleation group (73\%) compared to the formal resection-group $(62 \%)$. This is explained by the high pancreatic fistula rate in the enucleation group (27\% grade $\mathrm{C}$ pancreatic fistula). However, the overall severity of complications was greater in the formal resection 
group compared with the enucleation group (Clavien-Dindo classes 4-5, 10\% vs $0 \%$ ). Although long-term morbidity was not accessed in our study, previous studies have shown enucleation superior to formal resection in terms of diabetes and exocrine pancreas insufficiency. ${ }^{12}$ Enucleated tumors were all WHO 2010 grade 1 or 2, with size between 10 and $23 \mathrm{~mm}$, and none of them recurred or caused mortality. These results indicate that enucleation is a viable option in these patients with acceptable morbidity. It has been suggested that tumors over $2 \mathrm{~cm}$ should be treated with formal oncologic resection. ${ }^{2}$

There are limitations in our study. First, and most important, the studied patients represent only the cases that were operated and no comparison was made to patients that underwent observation alone. The surgery was performed in a tertiary center, and no register of patients undergoing surveillance in referring hospitals exists. Thus, no conclusions can be made on the natural course of NF-PNETs, and it remains unclear whether surgery improves the survival of patients with small NFPNETs. The answer to this question would require a randomized, prospective study, but because of the rarity of this disease, this type of study would need a multicenter approach. Second, this is a small retrospective cohort study, which all its limitations. However, due to the rarity of the disease, this is a common problem in the field of PNETs.

In conclusion, the malignant potential of NFPNETs seems diverse with no clear cut-off criteria for size to distinguish between benign and malignant disease. Preoperative work-up should include symptoms, size, and possibly WHO 2010 grade if available. Surgical resection should be considered in patients with symptomatic small NF-PNETs, who are fit for surgery. Asymptomatic small NF-PNETs seem to follow a benign course, and might be best treated by close surveillance. Larger scale, preferably multicenter, studies would be needed to confirm these results.

\section{REFERENCES}

1. Kuo JH, Lee JA, Chabot JA. Nonfunctional pancreatic neuroendocrine tumors. Surg Clin North Am 2014;94: 689-708.

2. Birnbaum DJ, Gaujoux S, Cherif R, Dokmak S, Fuks D, Couvelard A, et al. Sporadic nonfunctioning pancreatic neuroendocrine tumors: prognostic significance of incidental diagnosis. Surgery 2014;155:13-21.

3. Rindi G, Arnold R, Bosman FT, Capella C, Kilmstra DS, Klöppel G, et al. Nomenclature and classification of neuroendocrine neoplasms of the digestive system. In: WHO Classification of Tumours of the Digestive System. 4th ed. Lyon, France: World Health Organization; 2010.

4. Fischer L, Bergmann F, Schimmack S, Hinz U, Prieß S, Müller-Stich BP, et al. Outcome of surgery for pancreatic neuroendocrine neoplasms. Br J Surg 2014;101:1405-12.

5. Rindi G, Klöppel G, Alhman H, Caplin M, Couvelard A, Herder WW, et al. TNM staging of foregut (neuro)endocrine tumors: a consensus proposal including a grading system. Virchows Arch 2006;449:395-401.

6. Janson ET, Sorbye H, Welin S, Federspiel B, Grønbæk H, Hellman P, et al. Nordic guidelines 2014 for diagnosis and treatment of gastroenteropancreatic neuroendocrine neoplasms. Acta Oncol 2014;53:1284-97.

7. Bassi C, Dervenis C, Butturini G, Fingerhut A, Yeo C, Izbicki J, et al. Postoperative pancreatic fistula: an international study group (ISGPF) definition. Surgery 2005;138: 8-13.

8. Wente MN, Bassi C, Dervenis C, Fingerhut A, Gouma DJ, Izbicki JR, et al. Delayed gastric emptying (DGE) after pancreatic surgery: a suggested definition by the International Study Group of Pancreatic Surgery (ISGPS). Surgery 2007;142:761-8.

9. Haynes AB, Deshpande V, Ingkakul $T$, Vagefi PA, Szymonifka J, Thayer SP, et al. Implications of incidentally discovered, nonfunctioning pancreatic endocrine tumors: short-term and long-term patient outcomes. Arch Surg 2011;146:534-8.

10. Kim MJ, Choi DW, Choi SH, Heo JS, Park HJ, Choi KK, et al. Surgical strategies for non-functioning pancreatic neuroendocrine tumours. Br J Surg 2012;99:1562-8.

11. Wong JM, Fulp WJ, Strosberg JR, Kvols LK, Centeno BA, Hodul PJ. Predictors of lymph node metastases and impact on survival in resected pancreatic neuroendocrine tumors: a single-center experience. Am J Surg 2014;208: $775-80$.

12. Cherif R, Gaujoux S, Couvelard A, Dokmak S, Vuillerme MP, Ruszniewski P, et al. Parenchyma-sparing resections for pancreatic neuroendocrine tumors. J Gastrointest Surg 2012;16:2045-55. 